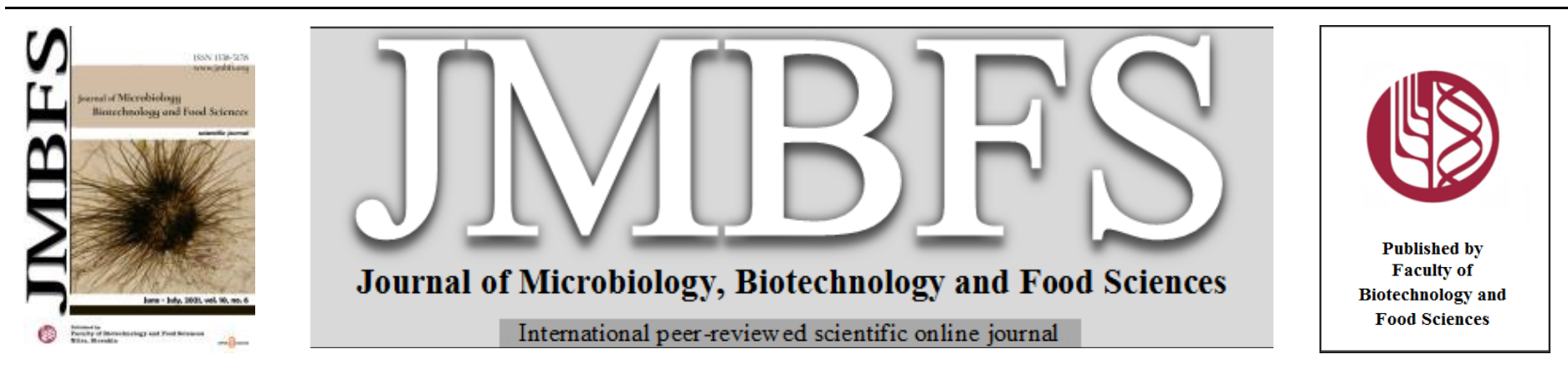

\title{
DEVELOPMENTAL TOXICITY OF ARISTOLOCHIC ACID IN DEVELOPING ZEBRAFISH (Danio rerio) EMBRYOS: CARDIOVASCULAR FAILURE AND INHIBITED CAUDAL FIN REGENERATION
}

\author{
Bangeppagari Manjunatha ${ }^{1,2}$, B. Sreevidya ${ }^{3}$, Sang Joon Lee ${ }^{2}$, Ke-Chun Liu ${ }^{1, *}$ \\ Address(es): \\ ${ }^{1}$ Key Laboratory for Drug Screening Technology of Shandong Academy of Sciences, Qilu University of Technology (Biology Institute of Shandong Academy of \\ Sciences), Jinan - 250103, PR China. \\ ${ }^{2}$ Center for Biofluid and Biomimic Research, Pohang University of Science and Technology (POSTECH), Pohang - 37673, South Korea \\ ${ }^{3}$ Narayana Medical College and Hospital, Nellore, Andhra Pradesh - 524003, India.
}

*Corresponding author: hliukch@sdas.org; manjubhargav2010@gmail.com

https://doi.org/10.15414/jmbfs.3160

ARTICLE INFO

Received 22. 5. 2020

Revised 19. 1. 2021

Accepted 21. 1.2021

Published 1. 6. 2021

Regular article

open ${ }_{\text {Aackess }}$

\begin{abstract}
The study employed a green fluorescent vascular endothelium transgenic line, Tg (VEGFR2: GFP), to evaluate the effects of aristolochic acid (AA) at various concentrations on cardiovascular and fin imperfections in developing zebrafish embryos. The findings reveal that the intersegmental vessels (ISVs), dorsal longitudinal anastomotic vessels (DLAVs), and fin regeneration in the embryos were attenuated and underdeveloped in comparison to the control at 1, 5, 10, 20, 30, and $40 \mu \mathrm{M} / \mathrm{L}$ concentrations of AA. At 48 hours post-fertilization (hpf), pericardial sac edema, yolk sac edema, and slight trunk curvature were detected in the surviving larvae at 10 and $20 \mu \mathrm{M} / \mathrm{L}$. These consequences were more pronounced at AA concentrations of 30 and $40 \mu \mathrm{M} / \mathrm{L}$. A gradual reduction in survival, hatching, heart rate, length, and diameter of ISVs and DLAVs was observed at 10, 20, 30, and $40 \mu \mathrm{M} / \mathrm{L}$ when the embryos were 48 hpf. In addition, the fin redevelopment of the zebrafish embryos was also constrained in the treatment groups. The embryos exhibited heartbeats; however, blood circulation was terminated in response to the additive effect of AA at 10 and $20 \mu \mathrm{M} / \mathrm{L}$ concentrations. Furthermore, at higher AA levels, the embryos did not hatch. Instead, they died. The present study verified that AA has a significant toxic effect on zebrafish embryos. These outcomes may add to the existing knowledge of the possible toxicological impacts of AA and pave the path toward the sustainable development of treatments that promote human health.
\end{abstract}

\section{INTRODUCTION}

Natural products represent significant reservoirs of uncharted additives that may benefit future drug discovery. China has a long history of using herbal-based treatments as remedies for various diseases. For instance, aristolochic acid (AA) and nitrophenanthrene carboxylic acid are commonly found in Asarum or Aristolochia, a plant that belongs to the Aristolochiaceae family. The essential AA derivatives found within plant extract of Aristolochia species are Aristolochic acids I (AAI) and II (AAII), both of which are used in herbal therapies. AA intake commonly alters kidney functioning in a process that is referred to as "Aristolochic Acid Nephropathy" (AAN) or "Chinese Herbs Nephropathy." AA toxins in food have also been found to cause a chronic renal disease known as Balkan Endemic Nephropathy (Jadot et al., 2017). However, despite the apparent toxic nature of AAI and AAII, many of these acids continue to be used in herbal treatments (Vaclavik et al., 2014; Yang et al., 2014). Specifically, Aristolochia plant extracts are employed to treat arthritis, gout, and persistent wounds (Arlt et al., 2002).

In the early 1990s, kidney failure was observed in almost 100 women who had attended a Belgian weight-loss clinic and taken a weight-loss supplement that contained AA-derived herbs (Vanherweghem, 1998; Ioset et al., 2003). Similar cases were also observed in Taiwan and elsewhere in Asia, with studies revealing that AA consumption resulted in both kidney failure and liver cancer (Hoang $\boldsymbol{e t}$ al., 2013; Ng et al., 2017). Subsequently, additional reports of the use of a more reliable method (LC-MS/MS) to quantify AA-derived DNA adducts (Yun $\boldsymbol{e}$ t al., 2012) that is also applicable to difficult samples, such as paraffin-embedded tissue, and implementation for high throughput (Yun et al., 2017). In more recent times, Bastek $\boldsymbol{e}$ t al. (2019) utilized a sensitive and reliable UPLC-MS/MS technique to quantify 7-(deoxyadenosine-N6-yl) aristolactam I (dA-AAI) adduct in DNA from human renal cell systems and tissue samples. Researchers have also used primary human hepatocytes and renal proximal tubule epithelial cells in an organ-on-a-chip microphysiological system, aristolactam-N-sulfated ester (AL-I $\mathrm{N}-\mathrm{OSO} 3$ ) as the key intermediate that would be formed in the hepatocytes and transported to the renal cortex to induce overt cytotoxicity and, likely, the formation of dA-AAI adducts (Chang et al., 2017). In addition, Schiffer $\&$ Friederich-Persson (2017) provided insights into the predominant pathways of AA metabolism and, thus, AAI DNA adduct formation and AAI-induced toxicity. Interestingly, both herbal extracts that can enhance or suppress angiogenesis and natural angiogenesis inhibitors and enhancers have attracted significant interest (Han et al., 2012). The process of angiogenesis and physiologic and pathologic circumstances have been examined in depth in a range of studies (Carmeliet \& Jain, 2011; Carmeliet, 2005; Presta et al., 2005; Folkman \& Shing, 1992; Melani \& Weinstein, 2010; Adams \& Alitalo, 2007; Coultas et al., 2005).

Factors like vascular endothelial cell growth factors (VEGF), fibroblast growth factors (FGF), ephrin receptors, and angiopoietins, to name a few, are wellpreserved and perform the same function in zebrafish (Liang et al., 2001; Lyons et al., 1998; Fouquet et al., 1997; Hanahan, 1997). Over the last 15 years, the zebrafish's caudal fin has been found to represent a useful model for the purposes of studying the process of regeneration after human injury or amputation (Akimenko et al., 2003; Poss et al., 2003; Becerra et al., 1996; Santamaria \& Becerra, 1991). However, there remains a lack of genetic and functional comprehension of fin regeneration (Padhi et al., 2004; Nechiporuk et al., 2003). The zebrafish is regarded as one of the leading vertebrate models for studying human diseases (Zon \& Peterson, 2005) that result from herbal remedies. However, there is a need to perform toxicity studies to confirm the extent to which the findings of such studies can inform the development of medical therapies. In view of this, the purpose of this study was to assess the correlation between AA and cardiovascular and fin defects in developing zebrafish embryos. It is anticipated that the findings of this study may add to the existing comprehension of the toxicologic consequences of $\mathrm{AA}$ and contribute to the development of sustainable drugs that are safe for human use. 


\section{MATERIALS AND METHODS}

\section{Zebrafish maintenance}

$\mathrm{AB}$ strain and Tg (VEGFR2: GFP) zebrafish (Danio rerio) were acquired from Dr. Jingwei Xiong of Harvard Medical School (Boston, Massachusetts, USA) and subsequently managed according to the standard protocols (Westerfield, 1995). Adult female and male zebrafish were separated in a breeding tank separated by a mesh panel. Natural mating commenced when the screen was removed, and the tank was illuminated. The resulting embryos were collected within half an hour of spawning. After undergoing rinsing three times, the healthy embryos were transferred to petri dishes or a sizable container containing embryo medium and cultured at $28^{\circ} \mathrm{C}$. The techniques for zebrafish culture, embryo collection, and fluorescence observation suggested by Chen, (2007) were adhered to. The development stages of the embryos were classified according to the standard procedure set out by Kimmel et al. (1995). All animal tests that were performed in this research were implemented in accord with the regulations published by the Regional Animal Ethics Committee.

\section{Chemical exposure}

Aristolochic acid (AA; Sigma, C17H11NO7, molecular weight $341.27 \mathrm{~g} / \mathrm{mol}$ ) was dissolved in DMSO (Dimethyl sulfoxide) at the appropriate concentrations. For the purposes of the toxicity tests, the AA solutions were diluted with embryo medium (Westerfield, 1995). The healthy embryos were selected under a stereomicroscope (Olympus SZX16; Tokyo, Japan) and moved into a 24-well microplate with 10 embryos per well in $2 \mathrm{~mL}$ solution. Embryos at 24 hours postfertilization (hpf) were exposed to various concentrations $(1,5,10,20,30$, and 40 $\mu \mathrm{M} / \mathrm{L}$ ) of $\mathrm{AA}$, and the controls were incubated in embryo medium comprising DMSO only (mock-treated control).

\section{Toxicity and morphology in zebrafish}

The toxicological, morphological, and developmental impacts of AA were evaluated. The zebrafish embryos were subsequently transferred into embryo medium and incubated for a further 24 hours. Images were then taken using a MacroView Fluorescence Microscope with Hamamatsu C9300-221 high-speed digital CCD camera (Olympus MVX10, Japan) to assess blood vessel development at $48 \mathrm{hpf}$. Tg (VEGFR2: GFP) transgenic zebrafish with fluorescent blood vessels was utilized to expedite image evaluation. The morphological phenotypes, hatching rate, intersegmental vessel (ISV), mortality rate, heart rate, and dorsal longitudinal anastomotic vessel (DLAV) defects of the embryos were assessed. The length and diameter of the ISVs and DLAVs of the zebrafish embryos were also evaluated using NIH ImageJ software (NIH). All tests were duplicated three times and performed in accordance with national and institutional regulations for the safeguarding of animal welfare and human subjects.

\section{Fin amputation in larval zebrafish}

Anaesthetized embryos aged $1 \mathrm{dpf}$ posterior to the end of the notochord with a 26-gauge needle tip with $100 \mathrm{ppm}$ of eugenol and transected the tail primordium. Ten embryos were each placed into a single well within a 24 -well plate. Each well contained $2 \mathrm{~mL}$ embryo medium. A pre-determined amount of the drug was placed into the embryo medium. Embryos at $24 \mathrm{hpf}$ were exposed to 1 and 5 $\mu \mathrm{M} / \mathrm{L}$ of $\mathrm{AA}$, and the controls were incubated in embryo medium comprising DMSO (mock-treated control). After the embryos had undergone 3 days of incubation, ImageJ software was used to assess the degree of fin regeneration from the larvae. The notochord was used as the point of reference.

\section{Statistical analysis}

All statistical assessments were performed using the SPSS Version 12, and the GraphPad Prism5 (GraphPad Software Inc., USA). All data presented in the graphs were described as mean $\pm \mathrm{SD}$, and the data were analyzed using one-way ANOVA. Values of ${ }^{*} p<0.01$ were considered to be of statistical significance.

\section{RESULTS}

\section{Morphological studies}

AA was added at various concentrations $(1,5,10,20,30$, and $40 \mu \mathrm{M} / \mathrm{L})$ to 24 well cell culture plates and analyzed on 24 -hpf embryos after 72 hours. The rates of survival and mortality were recorded. A digital camera and stereomicroscope were used to view any morphological and functional anomalies of the embryos at 48 and $72 \mathrm{hpf}$. The embryos treated with 30 and $40 \mu \mathrm{M} / \mathrm{L}$ or higher AA concentrations exhibited a significantly lower hatching rate than those placed in the control solution (Fig. 3B). The median effective concentration $\left(\mathrm{EC}_{50}\right)$ of AA for mortality was $16.05 \mu \mathrm{M} / \mathrm{L}$ with $95 \%$ confidence limits (lower $11.08 \mu \mathrm{M} / \mathrm{L}$; upper $20.24 \mu \mathrm{M} / \mathrm{L}$ ) for the 96 -hpf embryos. Slow blood circulation in the entire body and a median impact on hatching was exhibited within the specimens that were subjected to $10,20,30$, and $40 \mu \mathrm{M} / \mathrm{L}$ of AA at $48 \mathrm{hpf}$. At $72 \mathrm{hpf}$, blood circulation terminated in the whole body of the embryo; however, the embryos in the 10 and $20 \mu \mathrm{M} / \mathrm{L}$ treated groups continued to exhibit a heartbeat, while those in the 30 and $40 \mu \mathrm{M} / \mathrm{L}$ treated groups did not. At $48 \mathrm{hpf}$, embryo pericardial sac edema, yolk sac edema, and slight trunk curvature were seen in the surviving larvae in the 10 and $20 \mu \mathrm{M} / \mathrm{L}$ concentrations of AA (Fig. 1B and D), but yolk sac edema, heavy trunk curvature, and reduced cardiac edema were observed among those in 30 and $40 \mu \mathrm{M} / \mathrm{L}$ concentrations (Fig. $1 \mathrm{~F}$ and $\mathrm{H}$ ). At $72 \mathrm{hpf}$, the cardiac edema of the embryos in the 10 and $20 \mu \mathrm{M} / \mathrm{L}$ concentrations of AA decreased and heavy trunk curvature was seen (Fig. 1C and E). At 48 hpf, only a few of the embryos in the 30 and $40 \mu \mathrm{M} / \mathrm{L}$ concentrations of AA hatched (Fig. 1G). In 10 20, 30, and $40 \mu \mathrm{M} / \mathrm{L}$ at $48 \mathrm{hpf}$, embryo survival, hatching, and heart rate gradually reduced compared to the control (Fig. 3A, B and C).

\section{AA effects on DLAV and ISVs development}

The above hypothesis was also examined on zebrafish as an animal model method. AA partly constrained angiogenesis when utilized at an intensity of 10 20,30 , and $40 \mu \mathrm{M} / \mathrm{L}$ over 24 hours (Fig. 2A, B, C, D, and E). The length of the ISVs and DLAVs of the embryos in the control group at $48 \mathrm{hpf}$ was $1596.09 \pm$ 13.9 and $1217.9 \pm 12.3 \mu \mathrm{m}$. The AA-exposed embryos exhibited significantly shorter ISVs of $641.01 \pm 8.79,563.57 \pm 17.5,338.41 \pm 17.16$, and $162.24 \pm 9.83$ at $10,20,30$, and $40 \mu \mathrm{M} / \mathrm{L}$ concentrations respectively and DLAVs of $1019.7 \pm$ $7.56,304.18 \pm 19.19,192.94 \pm 6.73$, and $144.63 \pm 6.09 \mu \mathrm{m}$ at $10,20,30$, and 40 $\mu \mathrm{M} / \mathrm{L}$ dosages respectively (Fig. 3D and E). The average ISV diameters of the AA-treated embryos were $12.62 \pm 0.36,10.33 \pm 0.41,6.26 \pm 0.41$, and $3.76 \pm$ 0.65 at $10,20,30$, and $40 \mu \mathrm{M} / \mathrm{L}$ dosages respectively, and the average diameters of the DLAVs were $5.9 \pm 0.72,4.8 \pm 0.66,2.9 \pm 0.52,1.4 \pm 0.22 \mu \mathrm{m}$ at 10,20 , 30 , and $40 \mu \mathrm{M} / \mathrm{L}$ dosages respectively. The average ISV and DLAV diameters of the control embryos were $15.24 \pm 0.14$ and $8.45 \pm 0.28 \mu \mathrm{m}$. A comparison of the ISV and DLAV values is presented in Fig. 3F and G. We observed that AA exposure at $10 \mu \mathrm{M} / \mathrm{L}$ resulted in only minor deficiency in terms of the formation of DLAV and ISV. However, at higher concentrations, AA exposure led to serious deficiencies in ISV and DLAV development in most embryos (Fig. 3D, E, F, and G). This impact was more evident at higher doses $(>30 \mu \mathrm{M} / \mathrm{L})$, though acute deformities were also noted. The severe deformity observed can be ascribed to the contamination effect of AA at higher intensities.

\section{AA inhibits caudal fin regeneration}

As larval fin regeneration represents an avascular process, the assay was performed in larval and adult zebrafish to distinguish between the effects of drugs on cell proliferation and angiogenesis. AA dose-dependent inhibition of fin regeneration in larval (Fig. 3H; Fig. 4A, B and C) and adult zebrafish was measured 3 days post-amputation (dpa). The larval fish in the control group regenerated $331.45 \pm 7.4 \mu \mathrm{m}$ of their initial fin length at $3 \mathrm{dpa}$. The level of fin regeneration observed among the specimens treated with $1 \mu \mathrm{M} / \mathrm{L}$ of AA fell to $100.72 \pm 2.8 \mu \mathrm{m}(\mathrm{p}<0.01)$ while that observed among those treated with $5 \mu \mathrm{M} / \mathrm{L}$ of AA fell to $51.75 \pm 3.5 \mu \mathrm{m}$ of the initial fin length $(\mathrm{p}<0.01)$.
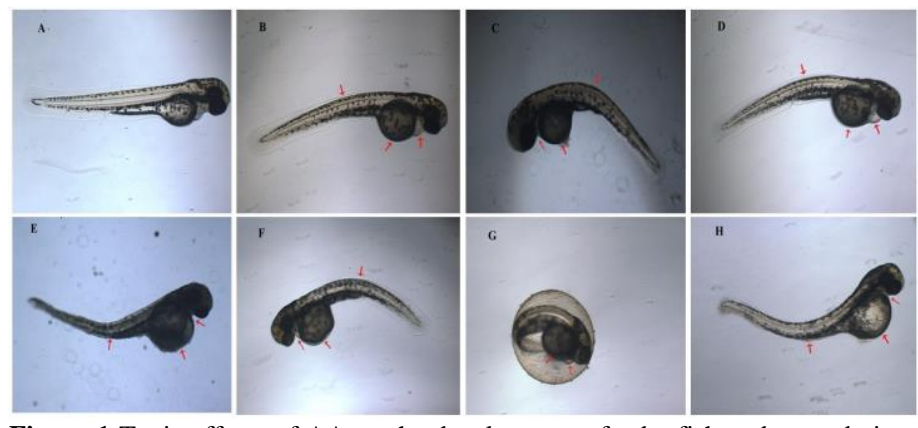

Figure 1 Toxic effects of AA on the development of zebrafish embryos obvious morphological abnormalities (arrows). (A) Normal embryo at 48 hpf. (B) $48 \mathrm{hpf}$ embryo with pericardial-sac edema, yolk-sac edema and light trunk curvature with $10 \mu \mathrm{M}$ AA. (C) 72 hpf embryo with pericardial-sac edema, yolk-sac edema and high trunk curvature with $10 \mu \mathrm{M}$ AA. (D) 48 hpf embryo with pericardial-sac edema, yolk-sac edema and trunk curvature with $20 \mu \mathrm{M}$ AA. (E) $72 \mathrm{hpf}$ embryo with yolk-sac edema, high trunk curvature and reduced pericardial-sac edema with $20 \mu \mathrm{M}$ AA. (F) 48-hpf embryo with yolk-sac edema, high trunk curvature and reduced pericardial-sac edema with $30 \mu \mathrm{M}$ AA. (G) $48 \mathrm{hpf}$ no hatched embryo with pericardial-sac edema, yolk-sac edema and high trunk curvature with $30 \mu \mathrm{M}$ AA. (H) 48 hpf embryo with yolk-sac edema, high trunk curvature and reduced pericardial-sac edema with $40 \mu \mathrm{M}$ AA. No significant differences were observed at $1,5 \mu \mathrm{M} / \mathrm{L}$ AA 


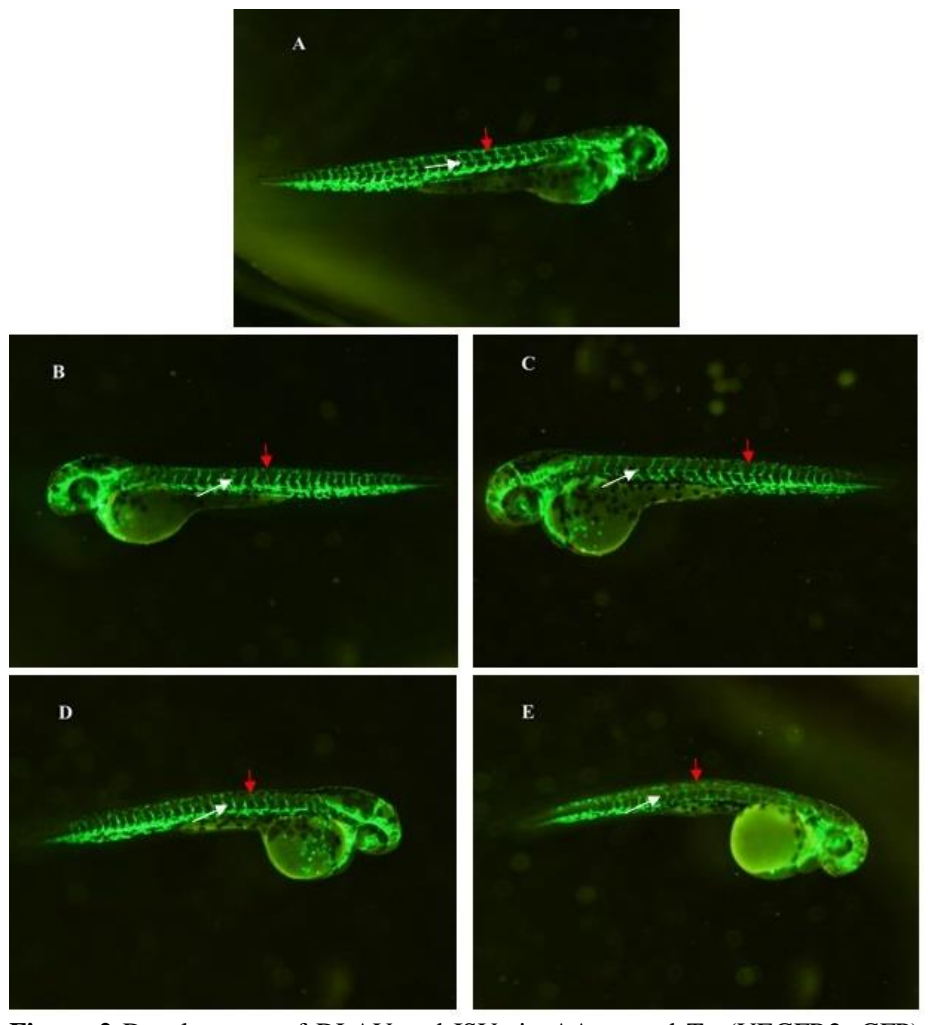

Figure 2 Development of DLAV and ISVs in AA-treated Tg (VEGFR2: GFP) embryos. (A) Normal embryo at $48 \mathrm{hpf}$ (B, C, D and E) 48 hpf embryo with DLAV and ISVs defects embryos were treated with 10, 20, 30 and $40 \mu \mathrm{M}$ AA. GFP fluorescent signal of the DLAV and ISVs of the transgenic zebrafish embryos was analyzed by fluorescent microscopy. ISVs are indicated by the white arrow. DLAV are indicated by the red arrow. No significant differences were observed at $1,5 \mu \mathrm{M} / \mathrm{L}$ AA.
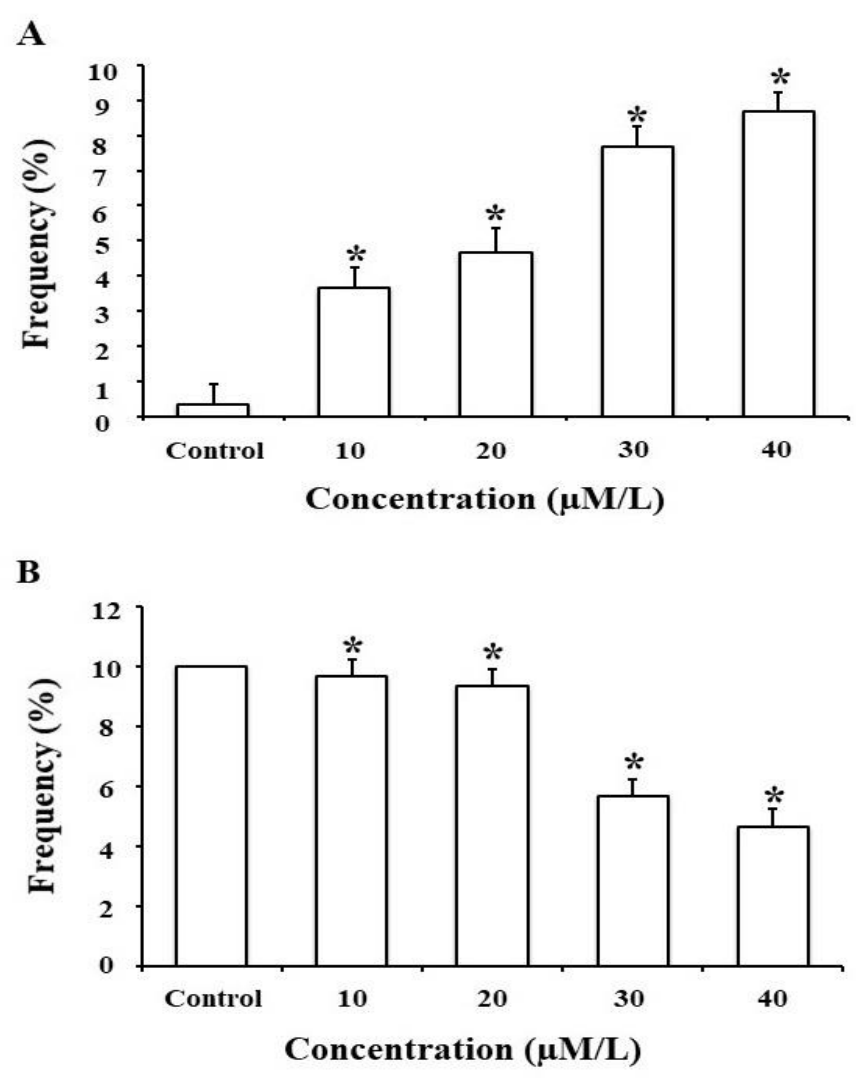
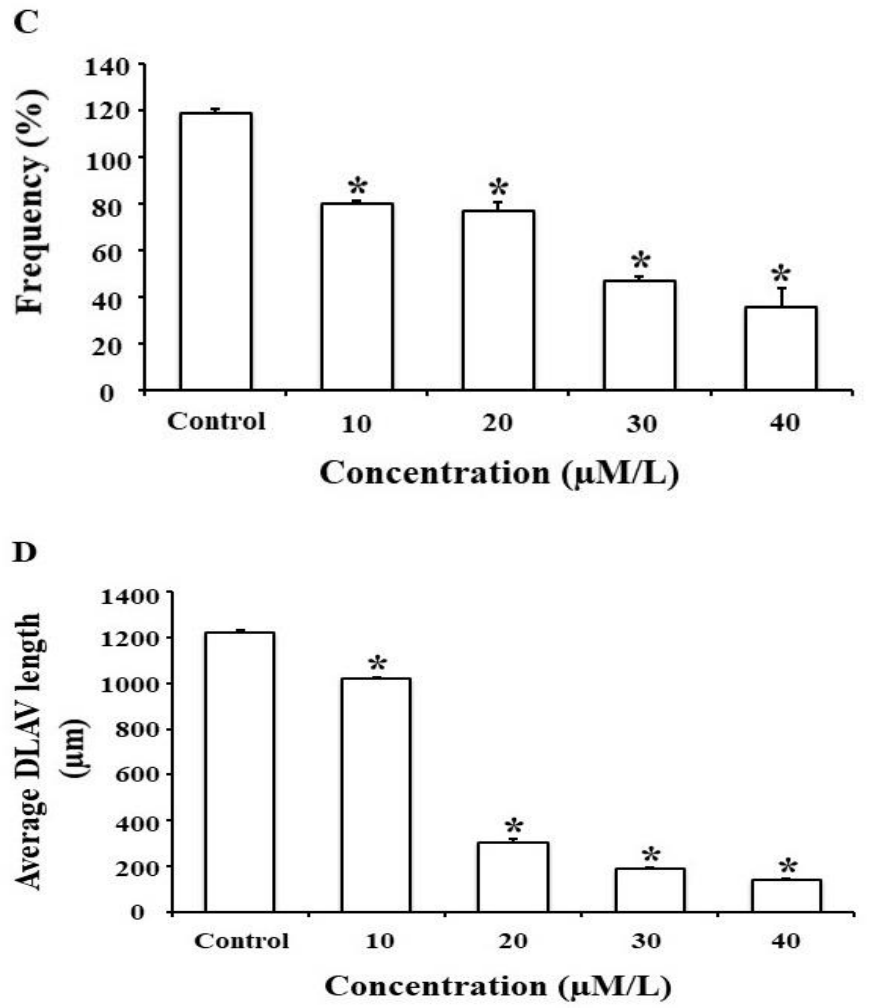

$\mathbf{E}$
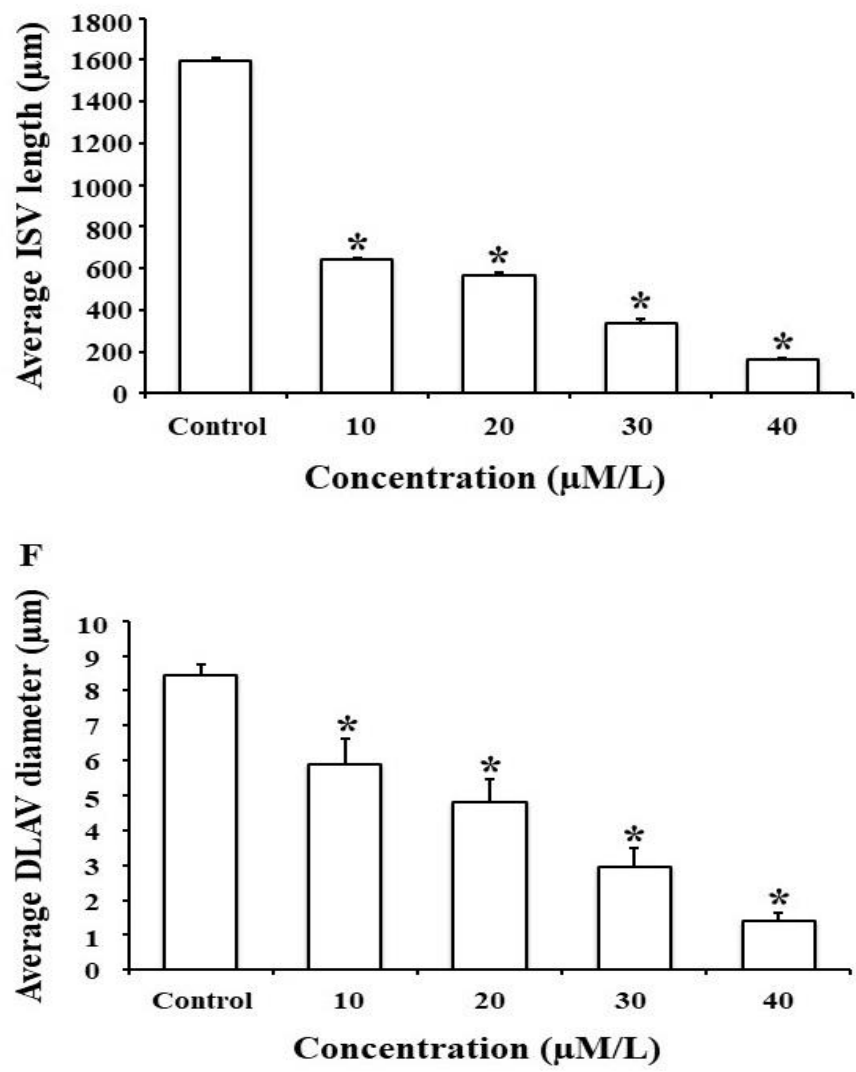
G

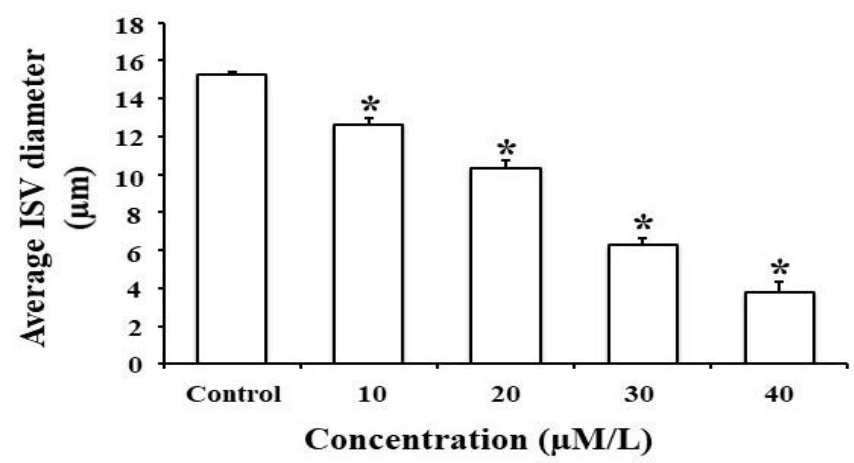

$\mathbf{H}$

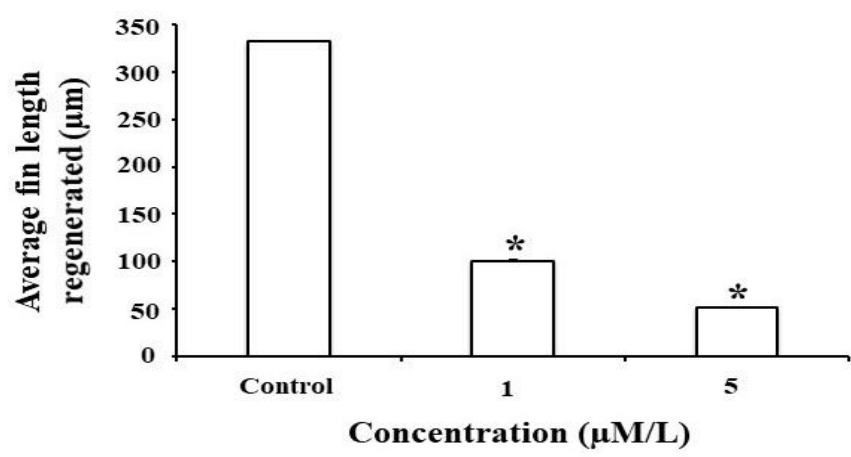

Figure 3 Effects of AA in terms of zebrafish mortality rate, hatching rate, heart rate, intersegmental vessels (ISVs), dorsal longitudinal anastomotic vessels (DLAV) and fin regeneration at $48 \mathrm{hpf}$. (A) A significant increase in mortality was observed in $10,20,30$ and $40 \mu \mathrm{M}$ of AA treated embryos. (B) The hatching rate significantly decreased in groups exposed to $10,20,30$ and $40 \mu \mathrm{M}$ of AA when compared to the control. (C) The heart rate significant difference between control and treated groups. (D, E, F and G) Summary of DLAV, ISVs length and diameter in $48 \mathrm{hpf}$ embryos control and exposed to AA at 10, 20,30 and $40 \mu \mathrm{M}$. (H) Summary of embryo caudal fin regeneration after three days exposed to AA $(1,5 \mu \mathrm{M})$. Results are expressed as mean \pm SD. Asterisks indicate significant differences compared to the control group $\left({ }^{*} p<0.01\right)$. All experiments were repeated three times. No significant differences were observed at $1,5 \mu \mathrm{M} / \mathrm{L}$ AA.
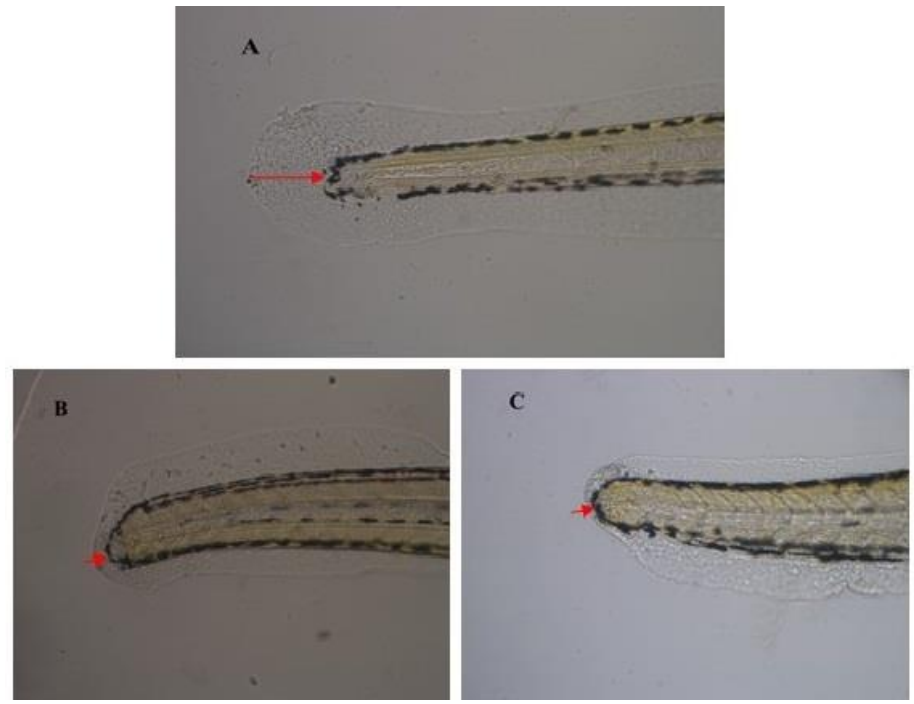

Figure 4 Summary of zebrafish caudal fin regeneration of larval zebrafish after three days exposed to AA. A) Normal larvae. B and C) Treated with 1 and $5 \mu \mathrm{M}$.

\section{DISCUSSION}

Angiogenesis is a regulated cellular process by which established vessels branch and invade tissues in reaction to normal or pathological stimulus (Staton $\boldsymbol{e t}$ al., 2009). Knowledge of the molecular systems that regulate angiogenesis is of clinical significance, and a variety angiogenesis assays are presently accessible for pro- and anti-angiogenic drug screening (Auerbach et al., 2003). Although endothelial cell models of apoptosis, proliferation, migration, and tube formation are prevalent due to their simple nature and throughput, these assays do not offer the biological intricacy of in vivo systems. The zebrafish model offers a mechanism for drug screening that not only exhibits the biological complexity of in vivo models but is also able to more readily replicate the capability of much higher-throughput screening than alternative popular animal models.

The embryonic vascular system develops due to both vasculogenesis and angiogenesis processes. Specifically, parachordal, intersegmental, and dorsal anastomotic vessels (ISVs, PAVs, and DLAVs, respectively) develop through a process of sprouting angiogenesis (Blum et al., 2008). Sprouting angiogenesis performs a fundamental role in the remodeling process by establishing new vascular loops that increase the vascular network. Scholars have identified several signaling pathways that promote angiogenic sprouting (Eilken \& Adams, 2010; Jakobsson et al., 2010)

Scholars have examined the implications of AA-induced toxicity across various animal models. The observations that have been published following previous studies strongly indicate that the most evident phenotype that results from AA exposure is angiogenesis. However, the way in which vascular development during embryogenesis is disrupted as a result of AA treatment remains unclear. In the current study, AA inhibited angiogenesis in rapidly developing blood vessels in the ISVs and DLAVs of zebrafish embryos between 24 and 48 hpf. Significant antiangiogenesis was observed in fully developed ISVs and DLAVs exposed to AA (data not shown). Exposure to AA during the 24-72 hpf period had a notable effect on the formation of new blood vessels and resulted in defects in ISVs and DLAVs that were not observed in the control group (Fig. 2). This indicates that AA administration stimulates vascular disruption and impedes the development of new blood vessels. Previous studies have found that developmental angiogenesis in the zebrafish embryo stimulates the formation of ISVs of the trunk (Cross $\boldsymbol{e t}$ al., 2003) and the SIV plexus (Serbedzija et al., 2000) represents a target for the screening of anti-angiogenic compounds. In these assays, low molecular weight compounds dissolved in fish water were assessed to determine the impact they had on the growth of new blood vessels that were promoted by the intricate network of endogenous, developmentally regulated signals.

Zebrafish caudal fin regeneration is frequently assessed to develop insights into the fundamental biological process by which tissues regenerate. Adult- and larval-stage zebrafish exhibit the same extraordinary ability to regenerate caudal fins in the aftermath of amputation (Mathew et al., 2006; Kawakami et al. 2004). AA impedes both life stages of caudal fin regeneration. The current study focused on the regeneration of zebrafish caudal fins following injuries that were inflicted during the larval stages of development (Fig. 4). However, minor variations in fin outgrowth were also seen during the late stages of regeneration between larval and adult animals. For instance, at $30 \mathrm{dpa}$, the regenerating cauda fin increased to approximately $95-98 \%$ of its original length in the young fish (4 months old), whereas the caudal fin's outgrowth was only $88 \%$ of the initial length in the older fish (18 months old). The processes that underpin unlimited caudal fin regeneration in adult zebrafish requires further investigation. We hypothesize that both epigenetic and genetic cues may be at play (Akimenko et al., 1995; Akimenko et al., 2003; Makino et al., 2005; Bouzaffour et al., 2009). The findings of the current study also indicated that embryo hatching was affected by the AA. Specifically, as the AA concentration increased, the hatching decreased. Comparable studies have examined the correlation between early embryonic exposure of Medaka fish and hatching (Hamm \& Hinton, 2000). At greater concentrations of pesticides, the eggs of Cyprinus carpio communis perished without hatching because the pesticides affected the enzyme activity responsible for hatching (Kaur \& Toor, 1977). Intriguingly, a rate of $30 \%$ and $50 \%$ hatching was seen in eggs taken from the mother fish exposed chronically to 2.6 and $1.3 \mathrm{mg} / \mathrm{L}$ for 30 days, respectively (Iqbal \& Mufti, 1992). Delayed or failed hatching due to the above-mentioned developmental deformities can be attributed to the effect of AA, which moderately or completely limits the developing embryo's or larvae's ability to break down the outer chorion and hatch. Likewise, the exposure of zebrafish embryos to alternative compounds have been found to result in failure to hatch (Wang et al., 2010; David \& Pancharatna, 2009; Strmac \& Braunbeck, 1999). The finding of the current study is aligned with these observations. Our findings clearly indicate that a low intensity of AA did not have any harmful effects on the early life stages of zebrafish and their hatching rate. However, a greater concentration (30 and 40 $\mu \mathrm{M} / \mathrm{L}$ ) of $\mathrm{AA}$ has the potential to impede the rate of hatching. The overall hatching success rates differed considerably amongst the various exposure groups (Fig. 3B). Moreover, variations were detected in either incidence of malformations or mortality between the treated and control embryos with differences in the dilutions of AA being of significance. Developmental abnormalities due to AA, such as the edema in the pericardial sac and lack of blood flow, may be the outcome of cardiotoxicity. Cardiotoxicity that results from AA exposure would have led to mortality, a delay in hatching, trunk curvature, pericardial edema, slow blood flow, yolk sac edema, and reduced heart rate. After $48 \mathrm{hpf}$, substantial disparities in the prevalence of edema were observed between the treated and control groups. Death was the primary outcome, which was the outcome of AA toxicity, while yolk-sac edema 
pericardial edema and were the most acute defects resulting from AA exposure (Fig. 1).

In the current study, the spatiotemporal mechanism of AA was explored for the first time by assessing its cardiovascular toxicity within a zebrafish model (Fig. 5). The drug metabolism systems of zebrafish have recently been shown to exhibit a high degree of functional similarity to that of mammals. As such zebrafish could be a useful model for cardiotoxicity studies. However, there is a requirement for further studies to better understand the relationships between the physiological systems that are regulated by AA.

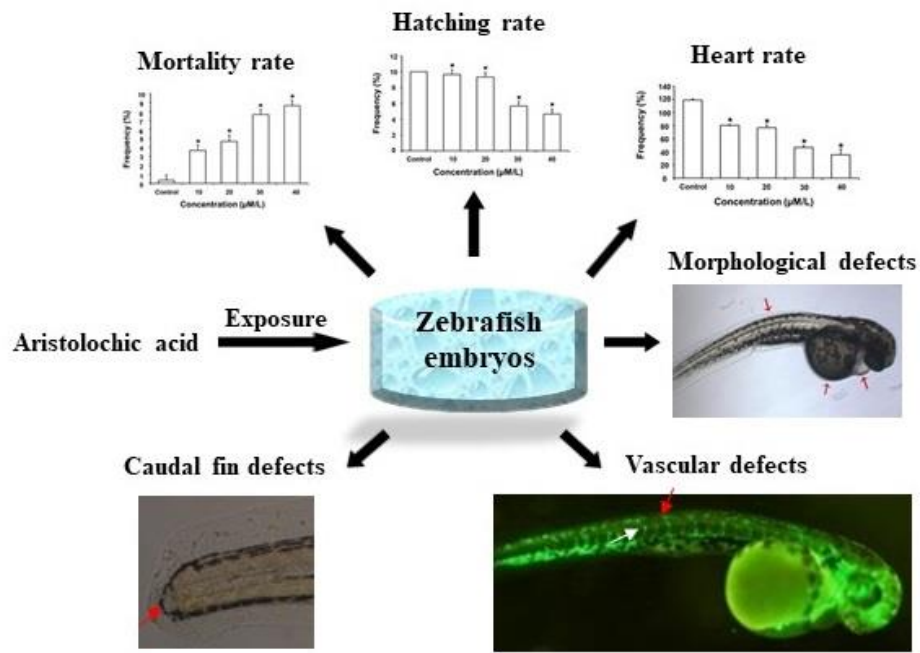

Figure 5 A schematic illustration of cardiovascular and caudal fin toxicity induced by AA in zebrafish (Danio rerio) model.

\section{CONCLUSION}

The findings of this study indicated that exposure to AA at concentrations of 1,5 , $10,20,30$, and $40 \mu \mathrm{M} / \mathrm{L}$ changed the physiological and morphological features of zebrafish embryos. We can conclude that AA influences the vascular development of zebrafish embryos, resulting in defects in ISVs and DLAVs. This finding supports the hypothesis that AA is a potent angiogenesis inhibitor. The results of this research clearly confirm that the zebrafish angiogenic model is a useful platform for screening anti-angiogenic drugs for human use on the basis that the drug metabolism systems of zebrafish exhibit functional similarity to that of mammals. The subtle changes induced by AA within this species is worth further investigation as part of efforts to better understand the impact AA has on the wellbeing of higher vertebrates.

Conflict of interest: The authors declare that they have no competing interest.

Acknowledgments: This study was supported by the National Natural Science Foundation of China (Grant No: 31400979) and Shandong Provincial Major Projects of Independent Innovation (2014ZZCX02105). The first-author greatly acknowledges the Korea Research Fellowship Program through the National Research Foundation of Korea (NRF) funded by the Ministry of Science and ICT (2016H1D3A1938252).

\section{REFERENCES}

Adams, R.H., Alitalo, K. (2007). Molecular regulation of angiogenesis and lymphangiogenesis. Nat. Rev. Mol. Cell Biol. 8: 464-478. https://doi.org/10.1038/nrm2183

Akimenko, M.A., Johnson, S.L., Westerfield, M., Ekker, M. (1995). Differentia induction of four msx homeobox genes during fin development and regeneration in zebrafish. Development. 121:347-357.

Akimenko, M.A., Mari-Beffa, M., Becerra, J., Geraudie J. (2003). Old questions, new tools, and some answers to the mystery of fin regeneration. Dev. Dyn. 226:190-201. https://doi.org/10.1002/dvdy.10248

Arlt, V.M., Stiborova, M., Schmeiser, H.H. (2002). Aristolochic acid as a probab le human cancer hazard in herbal remedies: a review. Mutagenesis. 17, 265-277. https://doi.org/10.1093/mutage/17.4.265

Auerbach, R., Lewis, R., Shinners, B., Kubai, L., Akhtar, N. (2003). Angiogenesis assays: a critical overview. Clin. Chem. 49: 32-40. https://doi.org/10.1373/49.1.32

Bastek, H., Zubel, T., Stemmer, K., Mangerich, A., Beneke, S., Dietrich, D.R (2019). Comparison of Aristolochic acid I derived DNA adduct levels in human renal toxicity models. Toxicology. 420, 29-38. https://doi.org.10.1016/j.tox.2019.03.013
Becerra, J., Junqueira, L.C.U., Bechara, I.J., Montes, G.S. (1996). Regeneration of fin rays in teleosts: a histochemical, radio-autographic, and ultrastructural study. Arch. Histol. Cytol. 59:15-35. https://doi.org/10.1679/aohc.59.15

Blum, Y., Belting, H.G., Ellertsdottir, E., Herwig, L., Lüders, F., Affolter, M (2008). Complex cell rearrangements during intersegmental vessel sprouting and vessel fusion in the zebra fish embryo. Dev. Biol. 316: 312-322. https://doi.org.10.1016/j.ydbio.2008.01.038

Bouzaffour, M., Dufourcq, P., Lecaudey, V., Haas, P., Vriz, S. (2009). Fgf and Sdf-1 pathways interact during zebrafish fin regeneration. PLoS One. 4:e5824 https://doi.org/10.1371/journal.pone.0005824

Carmeliet, P. (2005). Angiogenesis in life, disease and medicine. Nature. 438 (7070):932-936. https://doi.org.10.1038/nature04478

Carmeliet, P., Jain, R.K. (2011). Molecular mechanisms and clinical applications of angiogenesis. Nature. 473(7347):298-307. https://doi.org/10.1038/nature10144

Chen, Y.H., Chen, W.L., Wang, Y.H., Huang, M.Y., Chern, M.K. (2007) Spatiotemporal expression of zebrafish D-amino acid oxidase during early embryogenesis. Fish Physiol. Biochem. 33:73-80. https://doi.org/10.1007/s10695-006-9118-1

Coultas, L., Chawengsaksophak, K., Rossant, J. (2005). Endothelial cells and VEGF in vascular development. Nature. 438(7070): 937-945. https://doi.org.10.1038/nature04479

Cross, L.M., Cook, M.A., Lin, S., Chen, J.N., Rubinstein, A.L. (2003). Rapid analysis of angiogenesis drugs in a live fluorescent zebrafish assay. Arterioscler. Thromb. Vasc. Biol. 23: 911-912. https://doi.org/10.1161/01.ATV.0000068685.72914.7E

David, A., Pancharatna, K. (2009). Developmental anomalies induced by nonselective COX inhibitor (ibuprofen) in zebrafish (Danio rerio). Environ. Toxicol. Pharm. 27: 390-395. https://doi.org.10.1016/j.etap.2009.01.002

Eilken, H.M., Adams, R.H. (2010). Dynamics of endothelial cell behavior in sprouting angiogenesis. Curr. Opin. Cell Biol. 22: 617-625 https://doi.org.10.1016/j.ceb.2010.08.010

Folkman, J., Shing, Y. (1992). Angiogenesis. J. Biol. Chem. 267: 10931-10934. Fouquet, B., Weinstein, B.M., Serluca, F.C., Fishman, M.C. (1997). Vessel patterning in the embryo of the zebrafish: guidance by notochord. Dev. Biol. 183:37-48. https://doi.org/10.1006/dbio.1996.8495

Hamm, J.T., Hinton, D.E. (2000). The role of development and duration of exposure to the embryotoxicity of diazinon. Aquat. Toxicol. 48: 403-418. https://doi.org/10.1016/S0166-445X(99)00065-X

Han, L., Yuan, Y., Zhao, L., He, Q., Li, Y., Chen, X., Liu, X., Liu, K. (2012). Tracking antiangiogenic components from Glycyrrhiza uralensis Frisch. based on zebrafish assays using high-speed countercurrent chromatography. J. Sep. Sci. 35: 1167-1172. https://doi.org.10.1002/jssc.201101031

Hanahan, D. (1997). Signaling vascular morphogenesis and maintenance. Science. 277: 48-50. https://doi.org.10.1126/science.277.5322.48

Hoang, M.L., Chen, C.H., Sidorenko, V.S., He, J., Dickman, K.G., Yun, B.H., Moriya, M., Niknafs, N., Douville, C., Karchin, R., Turesky, R.J., Pu, Y.S., Vogelstein, B., Papadopoulos, N., Grollman, A.P., Kinzler, K.W., Rosenquist, T.A. (2013). Mutational signature of aristolochic acid exposure as revealed by wholeexome sequencing. Sci. Transl. Med. 5:197ra02. https://doi.org.10.1126/scitranslmed.3006200

Ioset, J.R., Raoelison, G.E., Hostettmann, K. (2003). Detection of aristolochic acid in Chinese phytomedicines and dietary supplements used as slimming regimens. Food Chem. Toxicol. 41(1):29-36. https://doi.org.10.1016/s02786915(02)00219-3

Iqbal, J., Mufti, S.A. (1992). Effect of diazinon on egg hatchability in a fresh water teleost, Colisa fasciata. Proc. Pak. Congress Zool. 11: 231-238.

Jadot, I., Decleves, A.E., Nortier, J., Caron, N. (2017). An integrated view of aristolochic acid nephropathy: update of the literature. Int. J. Mol. Sci. 18(2), 297 https://doi.org.10.3390/ijms18020297

Jakobsson, L., Franco, C.A., Bentley, K., Collins, R.T., Ponsioen, B., Aspalter, I.M., Rosewell, I., Busse, M., Thurston, G., Medvinsky, A., Schulte-Merker, S. Gerhardt, H. (2010). Endothelial cells dynamically compete for the tip cell position during angiogenic sprouting. Nat. Cell Biol. 12: 943-953. https://doi.org.10.1038/ncb2103

Kaur, K., Toor, H.S. (1977). Toxicity of pesticides to embryonic stages of Cyprinus carpio communis L. Indian J. Exp. Biol. 15: 193-196.

Kawakami, A., Fukazawa, T., Takeda, H. (2004). Early fin primordia of zebrafish larvae regenerate by a similar growth control mechanism with adult regeneration Dev. Dyn. 231: 693-699. https://doi.org.10.1002/dvdy.20181

Kimmel, C., Ballard, W.W., Kimmel, S.R., Ullmann, B., Schilling, T.F. (1995) Stages of embryonic develo pment in the zebra fish. Dev. Dyn. 203, 253-310.

Liang, D., Chang, J.R., Chin, A.J., Smith, A., Kelly, C., Weinberg, E.S., Ge, R (2001). The role of vascular endothelial growth factor (VEGF) in vasculogenesis angiogenesis, and hematopoiesis in zebra fish development. Mech Dev. 108: 2943. https://doi.org.10.1016/S0925-4773(01)00468-3

Lyons, M.S., Bell, B., Stainier, D., Peters, K.G. (1998). Isolation of the zebrafish homologues for the tie 1 and tie-2 endothelium -specific receptor tyrosine kinases Dev Dyn. 212: 133-140. https://doi.org.10.1002/dvdy.1157 
Makino, S., Whitehead, G.G., Lien, C.L., Kim, S., Jhawar, P., Kono, A., Kawata, Y., Keating, M.T. (2005). Heat-shock protein 60 is required for blastema formation and maintenance during regeneration. Proc Natl Acad Sci USA. 102:14599-14604. https://doi.org/10.1073/pnas.0507408102

Mathew, L.K., Andreasen, E.A., Tanguay, R.L. (2006). Aryl hydrocarbon receptor activation inhibits regenerative growth. Mol. Pharmacol. 69: 257-265. https://doi.org.10.1124/mol.105.018044

Melani, M., Weinstein, B.M. (2010). Common factors regulating patterning of the nervous and vascular systems. Annu. Rev. Cell Dev. Biol. 26: 639665. https://doi.org/10.1146/annurev.cellbio.093008.093324

Nechiporuk, A., Poss, K.D., Johnson, S.L., Keating, M.T. (2003). Positiona cloning of a temperature-sensitive mutant emmental reveals a role for sly1 during cell proliferation in zebrafish fin regeneration. Dev. Biol. 258(2): 291-306. https://doi.org/10.1016/S0012-1606(03)00129-5

Ng, A.W.T., Poon, S.L., Huang, M.N., Lim, J.Q., Boot, A., Yu, W., Suzuki, Y., Thangaraju, S., Ng, C.C.Y., Tan, P., Pang, S.T., Huang, H.Y., Yu, M.C., Lee, P.H., Hsieh S.Y., Chang, A.Y., Teh, B.T., Rozen, S.G. (2017). Aristolochic acids and their derivatives are widely implicated in liver cancers in Taiwan and $\begin{array}{llll}\text { throughout Asia. Sci. Transl. Med. 912):eaan6446 } & \end{array}$ https://doi.org.10.1126/scitranslmed.aan6446

Padhi, B.K., Joly, L., Tellis, P., Smith, A., Nanjappa, P., Chevrette, M., Ekker, M., Akimenko, M.A. (2004). Screen for genes differentially expressed during regeneration of the zebrafish caudal fin. Dev. Dyn. 231: 527-541. https://doi.org.10.1002/dvdy.20153

Poss, K.D., Keating, M.T., Nechiporuk, A. (2003). Tales of regeneration in zebrafish. Dev. Dyn. 226: 202-210. https://doi.org/10.1002/dvdy.10220

Presta, M., Dell'Era, P., Mitola, S., Moroni, E., Ronca, R., Rusnati, M. (2005).

Fibroblast growth facto r/fibroblast growth factor receptor system in angiogenesis. Cytokine Growth Factor Rev. 16: 159-178. https://doi.org/10.1016/j.cytogfr.2005.01.004

Santamaria, J.A., Becerra, J. (1991). Tail fin regeneration in teleosts: cellextracellular matrix interaction in blastemal differentiation. J. Anat. 176: 9-21.

Schiffer, T.A., Friederich-Persson, M. (2017). Mitochondrial reactive oxygen species and kidney hypoxia in the development of diabetic nephropathy. Front Physiol. 8, 211. https://doi.org.10.3389/fphys.2017.00211

Serbedzija, G.N., Flynn, E., Willett, C.E. (2000). Zebrafish angiogenesis: a new model for drug screening. Angiogenesis. 3: 353-359. https://doi.org.10.1023/a:1026598300052

Staton, C.A., Malcolm, W.R., Brown, N.J. (2009). A critical analysis of current in vitro and in vivo angiogenesis assays. Int. J. Exp. Pathol. 90: 195-221. https://doi.org.10.1111/j.1365-2613.2008.00633.x

Strmac, M., Braunbeck, T. (1999). Effects of triphenyltin acetate on survival, hatching success, and liver ultrastructure of early life stages of zebrafish (Danio rerio). Ecotoxicol. Environ. Saf. 44: 25-39. https://doi.org/10.1006/eesa.1999.1781

Vaclavik, L., Krynitsky, A.J., Rader, J.I. (2014). Quantification of aristolochic acids I and II in herbal dietary supplements by ultra-high-performance liquid chromatography-multistage fragmentation mass spectrometry. Food Addit. Contam. Part A, 31, 784-791. https://doi.org.10.1080/19440049.2014.892215

Vanherweghem, J.L. (1998). Misuse of herbal remedies: The case of an outbreak of terminal renal failure in Belgium (Chinese herbs nephropathy). J. Altern. Complement. Med. 4, 9-13. https://doi.org/10.1089/acm.1998.4.1-9

Wang, S., Liu, K., Wang, X., He, Q., Chen, X. (2010). Toxic effects of celastrol on embryonic development of zebrafish (Danio rerio). Drug and Chemical Toxicology. 34(1): 61-65. https://doi.org.10.3109/01480545.2010.494664

Westerfield, M. (1995). The Zebrafish Book: A Guide for the Laboratory Use of Zebrafish (Danio rerio), third ed., University of Oregon Press, Eugene.

Yang, H.Y., Chen, P.C., Wang, J.D. (2014). Chinese herbs containing aristolochic acid associated with renal failure and urothelial carcinoma: A review from epidemiologic observations to causal inference. Biomed. Res. Int. 2014 569325. https://doi.org.10.1155/2014/569325

Yun, B.H., Rosenquist, T.A., Sidorenko, V., Iden, C.R., Chen, C.-H., Pu, Y.-S. Bonala, R., Johnson, F., Dickman, K.G., Grollman, A.P., Turesky, R.J. (2012) Biomonitoring of Aristolactam-DNA adducts in human tissues using ultraperformance liquid Chromatography/Ion-Trap mass spectrometry. Chem. Res. Toxicol. 25, 1119-1131. https://doi.org.10.1021/tx3000889

Yun, B.H., Xiao, S., Yao, L., Krishnamachari, S., Rosenquist, T.A., Dickman, K.G., Grollman, A.P., Murugan, P., Weight, C.J., Turesky, R.J. (2017). A rapid throughput method to extract DNA from formalin-fixed paraffin-embedded tissues for biomonitoring carcinogenic DNA adducts. Chem. Res. Toxicol. 30, 2130-2139. https://doi.org.10.1021/acs.chemrestox.7b00218

Zon, L.I., Peterson, R.T. (2005). In vivo drug discovery in the zebrafish. Nat.

Rev. Drug Discov. 4:35-44. https://doi.org.10.1038/nrd1606 